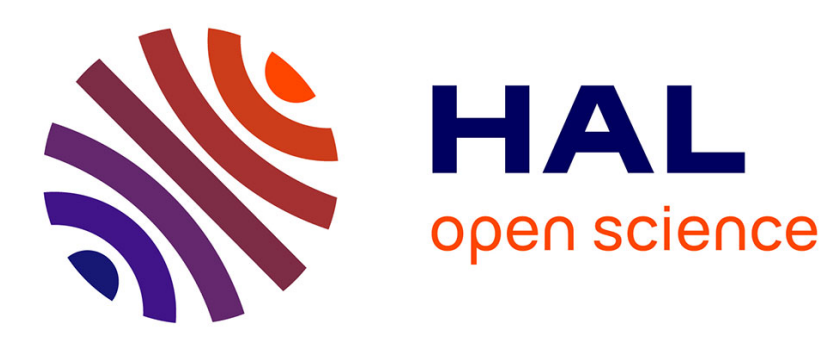

\title{
A Fully Discrete Approximation for Control Problems governed by Parabolic Variational Inequalities
}

\author{
Martine Bergounioux, Hasnaa Zidani
}

\section{To cite this version:}

Martine Bergounioux, Hasnaa Zidani. A Fully Discrete Approximation for Control Problems governed by Parabolic Variational Inequalities. [Research Report] RR-3940, INRIA. 2000. inria-00072710

\section{HAL Id: inria-00072710 \\ https://hal.inria.fr/inria-00072710}

Submitted on 24 May 2006

HAL is a multi-disciplinary open access archive for the deposit and dissemination of scientific research documents, whether they are published or not. The documents may come from teaching and research institutions in France or abroad, or from public or private research centers.
L'archive ouverte pluridisciplinaire HAL, est destinée au dépôt et à la diffusion de documents scientifiques de niveau recherche, publiés ou non, émanant des établissements d'enseignement et de recherche français ou étrangers, des laboratoires publics ou privés. 
INSTITUT NATIONAL DE RECHERCHE EN INFORMATIQUE ET EN AUTOMATIQUE

\section{A Fully Discrete Approximation for Control Problems governed by Parabolic Variational Inequalities}

Maïtine Bergounioux — Housnaa Zidani

$\mathbf{N}^{\circ} 3940$

Mai 2000

THÈME 4 



\title{
A Fully Discrete Approximation for Control Problems governed by Parabolic Variational Inequalities
}

\author{
Maïtine Bergounioux* , Housnaa Zidani ${ }^{\dagger \ddagger}$ \\ Thème 4 - Simulation et optimisation \\ de systèmes complexes \\ Projet MOCOA \\ Rapport de recherche $\mathrm{n}^{\circ} 3940$ - Mai $2000-23$ pages
}

\begin{abstract}
In this work we consider a numerical approximation of an optimal control problem governed by variational inequalities. We use a total discretization scheme: implicit Euler discretization with respect to the time variable and finite element method for the space variable, and give convergence results.
\end{abstract}

Key-words: Error estimates, approximations for control problems, state constraints, unbounded controls.

\footnotetext{
* Département de Mathématiques, UMR 6628 - MAPMO- Université d'Orléans Cedex 2, France. Email: Maitine.Bergounioux@labomath.univ-orleans.fr

† Département de Mathématiques, UMR 6628 - MAPMO- Université d'Orléans Cedex 2, France. Email: Hasna.Zidani@labomath.univ-orleans.fr

$\ddagger$ Action Mocoa, INRIA Rocqencourt, B.P 105, 78153 Le Chesnay,France, Email: Housnaa.Zidani@inria.fr
} 


\section{Discrétisation de problèmes de contrôle d'inéquations variationnelles paraboliques}

Résumé : Dans ce travail, nous considérons une approximation numérique pour un problème de contrôle optimal gouverné par une inéquation variationnelle parabolique. Nous établissons d'abord de nouvelles estimations d'erreur pour une discrétisation totale des équations paraboliques semilinéaires, et nous donnons ensuite des résultats de convergence pour la discrétisation du problème de contrôle optimal.

Mots-clés : Estimation d'erreur, contrôle optimal, contraintes sur l'état. 


\section{Introduction.}

1.1. Formulation of the problem. This paper deals with numerical approximation of optimal control problems for semilinear parabolic variational inequalities with controls in $L^{p}$ (not necessarily in $L^{\infty}$ ) and state constraints. The convergence analysis is the main objective.

Let $\Omega$ be a bounded convex open subset of $\mathbb{R}^{d}(d \geq 1)$, with Lipschitz boundary $\Gamma$. We fix $T>0$, and put $Q=\Omega \times] 0, T[$ and $\Sigma=\Gamma \times] 0, T[$.

Let $A$ be a second order uniformly elliptic operator:

$$
A y=-\sum_{j, k=1}^{d} D_{j}\left(a_{j k}(x) D_{k} y\right),
$$

where the coefficients $a_{j k} \in \mathcal{C}^{1+\beta}(\bar{\Omega})$ (with $\beta>0$ ) satisfy

$$
\sum_{j, k} a_{j k}(x) \chi_{j} \chi_{k} \geq m_{o}|\chi|^{2} \text { for all } \chi \in \mathbb{R}^{d} \text { and all } x \in \Omega
$$

with $m_{o}>0$. Consider the following parabolic variational inequality:

$$
\frac{\partial y}{\partial x}+A y+f(y)+\partial \kappa(y-\psi) \ni v \text { in } Q, \quad \frac{\partial y}{\partial n_{A}}+b y=0 \text { on } \Sigma, \quad y(0)=y_{o} \text { in } \Omega
$$

where $y_{o} \in H^{1}(\Omega) \cap \mathcal{C}(\bar{\Omega}), \psi \in L^{q}(Q)$ is defined everywhere on $Q, b \in \mathbb{R}^{+}, f: \mathbb{R} \rightarrow \mathbb{R}$, and the control variable $v$ is distributed. $\partial \kappa(y)(x, t)=\partial \kappa(y(x, t)), \partial \kappa(r)$ is the subdifferential of the function $\kappa$ at $r \in \mathbb{R}$ and $\kappa$ is the indicator function of $\mathbb{R}^{+}$:

$$
\kappa(r)=\left\{\begin{array}{cc}
0 & \text { if } r \geq 0 \\
+\infty & \text { else }
\end{array}\right.
$$

Define the following control constraint set:

$$
U_{a d}=\left\{v \in L^{q}(\Omega \times] 0, T[) \mid\|v\|_{q, \Omega \times] 0, T[} \leq M\right\},
$$

where $M$ is a fixed positive number. The control problem is defined by

$$
\left\{\begin{array}{l}
\operatorname{minimize} J(y, v)=\int_{0}^{T} \int_{\Omega} L(x, t, y) d x d t+\int_{0}^{T} \int_{\Omega} G(x, t, v) d x d t+\int_{\Omega} \ell(x, y(T)) d x \\
\text { subject to } v \in U_{a d}, \quad(y, v) \text { satisfies }(1.1), \quad F(x, t, y(x, t)) \leq 0 \quad \forall(x, t) \in \bar{\Omega} \times[0, T] .
\end{array}\right.
$$

We make the following additional assumptions: 
(H1) $f: \mathbb{R} \longrightarrow \mathbb{R}$ is Lipschitz on $\mathbb{R}$ and of class $\mathcal{C}^{1}$. Hereafter, we denote by $C_{o}, \widetilde{C}_{o} \in \mathbb{R}$ real numbers such that

$$
C_{o} \leq f^{\prime}(y) \leq \widetilde{C}_{o}, \quad \forall y \in \mathbb{R}
$$

(H2) $q>\frac{d}{2}+1$.

(H3) $y_{o} \in W_{o}^{2-\frac{1}{2 q}, q}(\Omega)\left(\subset \mathcal{C}(\bar{\Omega}) \cap W_{o}^{1, q}(\Omega)\right)$.

(H4) $\psi \in L^{q}(Q)$ is defined everywhere on $\bar{Q}$ and $y_{o}(x) \geq \psi(x, 0)$ for any $x \in \Omega$ (compatibility assumption for the initial condition).

(H5) $F, L, G: \Omega \times \mathbb{R} \times \mathbb{R} \longrightarrow \mathbb{R}$ are continuous. For any $(x, t) \in \Omega \times \mathbb{R}$, the function $F(x, t, \cdot)$ is Lipschitz continuous, and the function $G(x, t, \cdot)$ is convex.

(H6) $\ell: \Omega \times \mathbb{R} \longrightarrow \mathbb{R}$ is continuous. For any $x \in \Omega$, the function $\ell(x, \cdot)$ is Lipschitz.

The main goal of the present paper is to study a numerical approximation for $(\mathcal{P})$. Because of the variational inequality and state constraints this is delicate. First we use an idea based on the formulation of (1.1) as in [1] with a slackness variable and the regularity of its solution. Then we obtain a problem $(\widetilde{\mathcal{P}})$ equivalent to $(\mathcal{P})$, with constraints on both the control variable and state variable as coupled state/control constraints. We cannot avoid a first approximation of $(\widetilde{\mathcal{P}})$ that allows to relax some constraints. Otherwise, we would not be able to prove that the discretized formulation of the problem has at least one solution. Then we study the discretization of the relaxed problem and we shall prove that the discretized solutions are "close enough" the continuous one.

The main difficulty arising in the numerical study of optimal control problems governed by parabolic equations, is related to the nature of the state equation $[10,11,19]$. Indeed, error discretization estimations (either with respect to the time variable or to the space variable) are often established under regularity assumptions of the time-derivative of the solution [9]. In the case of optimal control problems, we cannot suppose that these assumptions are satisfied since it may change the nature of the problem under consideration.

There are many works on parabolic equations discretization process. We just mention here the book of Thome [18], where error estimates are given for equations with smooth data. We also mention the papers of Choudury [6,7], where some optimal error estimates are given for linear parabolic equations with nonsmooth data and with Dirichlet boundary conditions.

Here we are interested in the full discretization of a semilinear parabolic equation with nonsmooth data and with Robin-type boundary condition. We derive an error estimate in $L^{\infty}\left(L^{2}\right)$-norm (see theorem 3.2). For this, we use some technics similar to the ones already used by Nochetto-Verdi [13].

Notation . We denote by $Q$ the cylinder $\Omega \times] 0, T$ and by $\Sigma$ the lateral surface $\Gamma \times] 0, T[$. For any $s$ such that $1 \leq s \leq \infty$, the norms in the spaces $L^{s}(\Omega), L^{\tau}(\Gamma), L^{s}(Q), L^{\tau}(\Sigma)$ are denoted by $\|\cdot\|_{s, \Omega},\|\cdot\|_{s, \Gamma},\|\cdot\|_{s, Q},\|\cdot\|_{s, \Sigma}$. The inner products of $L^{2}(\Omega)$ and $L^{2}(\Gamma)$ are denoted respectively by $\langle,\rangle_{\Omega}$ and $\langle,\rangle_{\Gamma}$ while $\langle,\rangle_{H^{1}}$ denotes the canonical duality pairing between $H^{1}(\Omega)$ and $\left(H^{1}(\Omega)\right)^{\prime}$. 
The Hilbert space

$$
W\left(0, T ; H^{1}(\Omega),\left(H^{1}(\Omega)\right)^{\prime}\right)=\left\{y \in L^{2}\left(0, T ; H^{1}(\Omega)\right) \mid \frac{d y}{d t} \in L^{2}\left(0, T ;\left(H^{1}(\Omega)\right)^{\prime}\right)\right\}
$$

endowed with its usual norm, will be denoted by $W(0, T)$. The space $W^{2,1, q}(Q)$ is the usual space:

$$
W^{2,1, q}(Q)=\left\{y \in L^{q}(Q) \mid \frac{\partial y}{\partial t}, D_{x} y, D_{x x}^{2} y \in L^{q}(Q)\right\} .
$$

In the sequel, we denote by $C_{i}$, for $i \in \mathbb{N}-\{0\}$, generic constants occuring in the estimates given in propositions.

1.2. Preliminaries. The weak solution of (1.1) associated to $v$ is defined as the function $y \in W(0, T)$ satisfying $y(0)=y_{o}$, and

$$
\begin{gathered}
\int_{0}^{T}\left\langle\frac{\partial y}{\partial t}(t), y(t)-\varphi(t)\right\rangle_{H^{1}} d t+\int_{Q} \sum_{j, k} a_{j k}(x) D_{k} y D_{j}(y-\varphi) d x d t+\int_{Q} f(y)(y-\varphi) d x d t \\
+\int_{\Sigma} b y(y-\varphi) d s d t \leq \int_{Q} v(y-\varphi) d x d t
\end{gathered}
$$

for any $\varphi \in \mathcal{K}$ where

$$
\mathcal{K}=\left\{\varphi \in L^{2}\left(0, T ; H^{1}(\Omega)\right) \mid \varphi \geq \psi \text { a.e. in } Q\right\} .
$$

TheOREm 1.1. Assume (H1)-(H4). For every $u \in L^{q}(Q)$, the variational inequality (1.1) admits a unique weak solution $y_{v}$ in $\mathcal{C}(\bar{Q}) \cap W^{2,1, q}(Q)$. Moreover, the following holds.

i) For every $v \in L^{q}(Q)$, there exists $\theta \in L^{q}(Q)$, such that $y_{v}$ is also the solution of:

$$
\begin{gathered}
\left\{\begin{aligned}
\frac{\partial y}{\partial t}+A y+f(y)=v+\theta & \text { in } Q, \\
\frac{\partial y}{\partial n_{A}}+b y=0 & \text { on } \Sigma, \quad \text { and } \\
y(0)=y_{o} & \text { in } \Omega,
\end{aligned}\right. \\
\left\{\begin{aligned}
y(x, t)-\psi(x, t) \geq 0 & \forall(x, t) \in Q, \\
\theta(x, t) \geq 0 & \text { a.e. }(x, t) \in Q, \\
(y(x, t)-\psi(x, t)) \theta(x, t)=0 & \text { a.e. }(x, t) \in Q,
\end{aligned}\right.
\end{gathered}
$$

ii) There exists $C_{1}=C_{1}\left(T, \Omega, d, q, C_{o}, m_{o},\left\|y_{o}\right\|_{\mathcal{C}(\bar{\Omega})}\right)>0$ such that, for any $(v, \theta) \in$ $L^{q}(Q) \times L^{q}(Q)$, the weak solution $y_{v \theta}$ of $(1.2 \mathrm{a})$ associated to $(v, \theta)$ satisfies:

$$
\left\|y_{v \theta}\right\|_{W^{2,1, q}(Q)}+\left\|y_{v \theta}\right\|_{\mathcal{C}(\bar{Q})} \leq C_{1}\left(1+\|v\|_{q, Q}+\|\theta\|_{q, Q}\right)
$$


iii) For every $\varepsilon, K>0$, there exist $\alpha>0, C_{1}^{\prime}=C_{1}^{\prime}\left(T, \Omega, d, q, C_{o}, m_{o},\left\|y_{o}\right\|_{\mathcal{C}(\bar{\Omega})}, \varepsilon, K, \alpha\right)>$ 0 such that, for any $(v, \theta) \in L^{q}(Q) \times L^{q}(Q)$, $y_{v \theta}$ belongs to $\mathcal{C}^{\alpha, \frac{\alpha}{2}}(\bar{\Omega} \times[\varepsilon, T])$ and obeys

$$
\left\|y_{v \theta}\right\|_{\mathcal{C}^{\alpha, \frac{\alpha}{2}}(\bar{\Omega} \times[\varepsilon, T])} \leq C_{1}^{\prime}
$$

if $\|v\|_{q, Q}+\|\theta\|_{q, Q} \leq K$.

Proof - The proof of the above results is similar to the one given in [1].

In addition we shall use the following compactness result.

Theorem 1.2. The mapping $(v, \theta) \mapsto y(v, \theta)$ where $y(v, \theta)$ is the solution of (1.2a), is sequentially continuous from $L^{q}(Q) \times L^{q}(Q)$, endowed with the weak- $L^{q}(Q) \times L^{q}(Q)$ topology, into $\mathcal{C}(\bar{Q})$ (strong topology).

Proof - Let $\left\{\left(v_{n}, \theta_{n}\right)\right\}_{n}$ be a convergent sequence for the weak- $L^{q}(Q) \times L^{q}(Q)$ topology, and let $(v, \theta)$ be the weak limit of $\left(v_{n}, \theta_{n}\right)_{n}$. For any $n \in \mathbb{N}$, we denote by $y_{n}$ the solution $y\left(v_{n}, \theta_{n}\right)$ of $(1.2 \mathrm{a})$ associated to $\left(v_{n}, \theta_{n}\right)$. From Theorem 1.1 , we know that the sequence $\left(y_{n}\right)_{n}$ is bounded in $C(\bar{Q}) \cap W^{2,1, q}(Q)$. Furthermore, for every $\varepsilon>0,\left(y_{n}\right)_{n}$ is bounded in a Hölder space on $\bar{\Omega} \times[\varepsilon, T]$. Then, there exists $y \in \mathcal{C}(\bar{Q}) \cap W^{2,1, q}(Q)$ such that $\left(y_{n}\right)_{n}$ converges to $y$ weakly in $W^{2,1, q}(Q)$ and strongly in $\mathcal{C}(\bar{Q})$. By direct calculations, we can check that $y$ is the solution of $(1.2 \mathrm{a})$ corresponding to $(v, \theta)$.

2. An equivalent problem to $(\mathcal{P})$. Using the previous result we may replace the state inequation by a system of equations involving a new control variable which is the Lagrange multiplier associated to the variational inequality. Therefore, the control problem $(\mathcal{P})$ turns to be a "standard" optimal control problem governed by a state equation and involving additional constraints on both the state and the control functions. More precisely, consider a new set of controls:

$$
\Theta_{a d}=\left\{\theta \in L^{q}(Q) \mid \theta \geq 0 \text { a.e. in } Q\right\} .
$$

With Theorem 1.1, we see that problem $(\mathcal{P})$ is equivalent to the following one $(\widetilde{\mathcal{P}})$ :

$$
\begin{aligned}
& \text { Minimize } J(y, v) \text { subject to : } \\
& \frac{\partial y}{\partial t}+A y+f(y)=v+\theta \text { in } Q, \quad \frac{\partial y}{\partial n_{A}}+b y=0 \text { on } \Sigma, \quad y(., 0)=y_{o} \text { in } \Omega \\
& F(x, t, y(x, t)) \leq 0, y(x, t) \geq \psi(x, t) \text { in } \bar{Q} \quad \text { ("Pure" state constraints) } \\
& (v, \theta) \in U_{a d} \times \Theta_{a d} \quad \text { ("Pure" control constraints) } \\
& \int_{Q}(y(x, t)-\psi(x, t)) \theta(t, x) d x d t=0 \quad \text { (Mixed State/Control integral constraints) }
\end{aligned}
$$


We are going to perform a numerical study of this problem rather than the previous (genuine) one. To discretize the problem $(\widetilde{\mathcal{P}})$ and get some convergence results, we need to bound the new control function (see [2] for example) to use compactness properties. Therefore we set

$$
V_{a d}=\left\{(v, \theta) \in U_{a d} \times \Theta_{a d} \mid\|\theta\|_{q, Q} \leq \widetilde{M}\right\},
$$

where $\widetilde{M}$ is a constant which is allowed to be very large and must be greater than the norm of the control $\bar{\theta}$ corresponding to an optimal solution of $(\mathcal{P}$ ) (as in [2]). From a numerical point of view this may be the largest constant allowed by the computer. The problem $(\mathcal{P})$ is still equivalent to $(\widetilde{\mathcal{P}})$ when we replace $(2.2 \mathrm{c})$ by:

$$
(u, \theta) \in V_{a d}
$$

In the sequel we do not care about the existence of an optimal solution to $(\mathcal{P})($ or $(\widetilde{\mathcal{P}})$ ): one can refer to [1]. Since we are interested in the numerical approximation of these problems we assume from now that such an optimal solution exists and we call it $(\bar{y}, \bar{v}, \bar{\theta})$.

3. Discretization of the state equation. We first give some results about the discretization of the following parabolic equation:

$$
\frac{\partial y}{\partial t}+A y+f(y)=g \quad \text { in } Q, \quad \frac{\partial y}{\partial n_{A}}+b y=0 \quad \text { in } \Sigma, \quad y(0)=y_{o} \quad \text { in } \Omega,
$$

where $g$ belongs to $L^{q}(Q)$. The weak solution $y$ of (3.1) belongs to $W^{2,1, q}(Q)$, and satisfies

$$
\frac{d}{d t}\langle y(t), \chi\rangle_{H^{1}}+\mathcal{A}(y(t), \chi)+\int_{\Omega} f(y(x, t)) \chi(x) d x+\int_{\Gamma} b y(s, t) \chi(s) d s=\int_{\Omega} g \chi(x) d x
$$

for every $\chi \in H^{1}(\Omega)$ and a.e. $\left.t \in\right] 0, T$ [. The bilinear form $\mathcal{A}$ is defined as follows

$$
\forall y, z \in H^{1}(\Omega) \times H^{1}(\Omega) \quad \mathcal{A}(y, z)=\int_{\Omega} \sum_{j, k} a_{j k}(x) D_{k} y(x) D_{j} z(x) d x .
$$

3.1. Discretization and approximating spaces. Now, we make the discretization process precise: we use a finite difference scheme for the time variable (implicit Euler method) and a finite element approximation for the space variable (in $\Omega$ ).

3.1.1. Grid for $\Omega$. Let $\left(\mathcal{F}_{h}\right)_{h}$ be a family of triangulations of $\bar{\Omega}$ into closed $d$-simplices. To any simplex $K \in \mathcal{F}_{h}$, we associate two parameters:

- $h_{K}:=\operatorname{diam}(K)$,

- $\rho_{K}:=\sup \{\operatorname{diam}(S) \mid S$ is a ball contained in $K\}$.

We suppose that $h=\max _{K \in \mathcal{F}_{h}} h_{K}$ and that $\left(\mathcal{F}_{h}\right)_{h}$ is regular in the following sense ([8], p. 132):

i) There exist two positive numbers $\eta, \gamma$ such that:

$$
\frac{h_{K}}{\rho_{K}} \leq \eta \quad \text { and } \quad \frac{h}{\rho_{k}} \leq \gamma \quad \text { for all } K \in \mathcal{F}_{h} \quad \text { and all } h>0 .
$$


ii) We set $\bar{\Omega}_{h}=\bigcup_{K \in \mathcal{F}_{h}} K, \Omega_{h}$ its interior (in general, $\Omega_{h} \neq \Omega$ ) and $\Gamma_{h}$ its boundary. We assume that $\bar{\Omega}_{h}$ is convex.

REMARK 3.1. Since $\Omega$ is bounded, the discretization parameter $h$ is necessarily less than some constant $h_{\Omega}$ which only depends on $\Omega$.

To every simplex $K \in \mathcal{F}_{h}$ dealing with the boundary, we associate a "curved" simplex $\tilde{K} \in \bar{\Omega}$ such that the $d$ interior faces to $\Omega$ correspond with the ones of $K$, and such that the $(d+1)-t h$ face is the part of $\Gamma$ limited by the $d$ other faces. We denote by $\widetilde{\mathcal{F}}_{h}$ the familly composed by these simplexes $\tilde{K}$ and the simplexes contained inside $\Omega$. Hence we have: $\bar{\Omega}=\bigcup_{K \in \widetilde{\mathcal{F}}_{h}} K$.

To any such triangulation $\widetilde{\mathcal{F}}_{h}$ we associate the finite dimensional following space

$$
\mathcal{Y}_{h}=\left\{z \in \mathcal{C}(\bar{\Omega}):\left.z\right|_{K} \text { is affine for any } K \in \widetilde{\mathcal{F}}_{h}\right\} .
$$

Let $\left\{x_{j}\right\}_{j=1}^{N_{e}}$ be the set of all nodes of $\widetilde{\mathcal{F}}_{h}$ on $\bar{\Omega}_{h}$. Let $\varphi_{j}($.$) be the basis function associated$ to the node $x_{j}\left(\varphi_{j} \in \mathcal{Y}_{h}, \varphi_{j}\left(x_{k}\right)=1\right.$ if $k=j, \varphi_{j}\left(x_{k}\right)=0$, if $\left.k \neq j\right)$.

In order to analyze the error we perform if we consider the approximation $y_{h}$ of the system (3.7) instead of the (exact) solution $y$ of (3.1), we consider the bilinear form $a(.,$.$) defined$ on $H^{1}(\Omega)$ by

$$
a(w, z):=\mathcal{A}(w, z)+C_{o}\langle w, z\rangle_{\Omega}+b\langle w, z\rangle_{\Gamma} \quad \forall w, z \in H^{1}(\Omega),
$$

where $C_{o}$ is the constant given in (H1). We can suppose, without loss of generality [14], that

$$
a(z, z) \geq \frac{m_{o}}{2}\|z\|_{H^{1}(\Omega)}^{2} \quad \text { for any } z \in H^{1}(\Omega) .
$$

Let $E_{h}$ be the operator of $H^{1}(\Omega)$ on $\mathcal{Y}_{h}$ which associates to any $z \in H^{1}(\Omega)$ the unique element $E_{h} z$ of $\mathcal{Y}_{h}$ such that for all $\chi \in \mathcal{Y}_{h}$ :

$$
\sum_{l, j=1}^{d} \int_{\Omega} a_{l j}(x) D_{j}\left(z-E_{h} z\right) D_{l} \chi d x+\int_{\Omega} C_{o}\left(z-E_{h} z\right) \chi d x+\int_{\Gamma} b\left(z-E_{h} z\right) \chi d s=0 .
$$

We observe that this operator satisfies:

$$
a\left(E_{h} z, \chi\right)=a(z, \chi) \quad \text { for any } \chi \in \mathcal{Y}_{h} \text { and any } z \in H^{1}(\Omega) .
$$

Therefore it is a projection operator and we have the following classical approximation results [6]:

Proposition 3.1. There exists $C_{2}>0$ such that for every $h<h_{\Omega}$, we have

$$
\begin{gathered}
\left\|E_{h}\left(z_{1}-z_{2}\right)\right\|_{H^{1}(\Omega)} \leq C_{2} h\left\|z_{1}-z_{2}\right\|_{H^{1}(\Omega)} \quad \forall z_{1}, z_{2} \in H^{1}(\Omega), \\
\left\|E_{h} z-z\right\|_{2, \Omega} \leq C_{2} h\|z\|_{H^{1}(\Omega)} \quad \forall z \in H^{1}(\Omega) .
\end{gathered}
$$


3.1.2. Grid for $\Gamma$. We denote $\mathcal{B}_{h}$ the triangulation of $\Gamma$, inducted by the triangulation $\widetilde{\mathcal{F}}_{h}$.

3.1.3. Partition of $[0, T]$. Let $N$ be a positive integer. We consider the uniform partition of $[0, T]$ defined by:

$$
\begin{gathered}
t_{o}=0<t_{1}<t_{2}<\cdots<t_{N}=T \\
t_{i}=i \tau \quad(i=0, \ldots, N), \text { where } \tau:=\frac{T}{N} .
\end{gathered}
$$

For any $i=1, \ldots, N$, we denote by $\chi_{i}$ the characteristic function of $\left.] t_{i-1}, t_{i}\right]$.

The exact value $y^{i}=y\left(\cdot, t_{i}\right)$ of the weak solution $y$ of $(3.2)$ at time $t_{i}(i=1, \ldots, N)$ will be approximated by:

$$
y_{h}^{i}(\cdot):=\sum_{j=1}^{N_{e}} Y_{j}^{i} \varphi_{j}(.) \in \mathcal{Y}_{h}, \quad j=1, . ., N_{e}
$$

where $Y_{j}^{i}=y\left(x_{j}, t_{i}\right)$ (we remark that $Y_{j}^{i}=y\left(x_{j}, t_{i}\right) \in \mathbb{R}$ is well defined since $y \in \mathcal{C}(\bar{Q})$.)

Now we derive the discrete analog of (3.2) by means of which we shall define the approximate solution:

$$
\left\{\begin{array}{l}
\text { For } i=1, \ldots, N \text { and for any } \varphi \in \mathcal{Y}_{h} \\
\left.\int_{\Omega} \frac{y_{h}^{i}-y_{h}^{i-1}}{\tau} \varphi d x+\mathcal{A}\left(y_{h}^{i}, \varphi\right)+\int_{\Omega} f\left(y_{h}^{i}\right) \varphi d x+\int_{\Gamma} b y_{h}^{i} \varphi d s=\frac{1}{\tau} \int_{t_{i-1}}^{t_{i}} \int_{\Omega} g(x, t) \varphi d x^{3} d z t^{7}\right) \\
y_{h}^{o}:=E_{h} y_{o} .
\end{array}\right.
$$

We prove that there exists exactly one (finite) family of functions $\left\{y_{h}^{i}\right\}_{i=1}^{N}$ of the form (3.6) satisfying (3.7).

THEOREM 3.1. The system (3.7) admits a unique solution.

Proof - The proof of this theorem is based on compactness and monotony arguments (see [17], Chapter 5).

From now, we set $\delta=(h, \tau)$ (space-step, time-step), and we define the "discretized" solution $y_{\delta}$ for (3.1) using:

$$
\left.\left.y_{\delta}(\cdot, t)=\sum_{i=1}^{N} \chi_{i}(t) y_{h}^{i}(\cdot) \text { on }\right] 0, T\right], \text { and } y_{\delta}(\cdot, 0)=y_{h}^{o} .
$$

The function $y_{\delta}$ belongs to $L^{\infty}(Q)$ and

$$
\int_{Q} y_{\delta}^{2}(x, t) d x d t=\sum_{i=1}^{N} \int_{t_{i-1}}^{t_{i}} \int_{\Omega}\left(y_{\delta}(x, t)\right)^{2} d x d t=\tau \sum_{i=1}^{N} \int_{\Omega} y_{h}^{i}(x)^{2} d x=\tau \sum_{i=1}^{N}\left\|y_{h}^{i}\right\|_{2, \Omega}^{2} .
$$

REMARK 3.2. In the scheme (3.7), the time discretization is the implicit regressive Euler discretization, which is known to be inconditionally stable ([12], p. 107). 


\subsection{Error estimates for state equation discretization .}

Lemma 3.1. (Stability result) There exists $C_{3}=C_{3}\left(\Omega, d, \sigma, C_{o}, m_{o},\left\|y_{o}\right\|_{H^{1}(\Omega)}, M, h_{\Omega}\right)>$ 0 such that for any $h \in] 0, h_{\Omega}[, \tau \in] 0,1\left[\right.$, and $g \in L^{q}(Q)$ satisfying $\|g\|_{q, Q} \leq M$, the solution $\left\{y_{h}^{i}\right\}_{i=0, \ldots, N}$ to (3.7) corresponding to $g$ satisfies:

$$
\tau \sum_{i=1}^{N}\left\|y_{h}^{i}\right\|_{H^{1}(\Omega)}^{2}+\max _{1 \leq i \leq N}\left\|y_{h}^{i}\right\|_{2, \Omega}^{2}+\sum_{i=1}^{N}\left\|y_{h}^{i}-y_{h}^{i-1}\right\|_{2, \Omega}^{2} \leq C_{3}
$$

Proof - We take $\varphi:=\tau y_{h}^{i}$ in (3.7) and sum from $i=1$ to $i=i_{o}\left(i_{o}\right.$ being a generic index in $\{1, . ., N\})$; we obtain

$$
\begin{gathered}
\sum_{i=1}^{i_{o}} \int_{\Omega}\left(y_{h}^{i}-y_{h}^{i-1}\right) y_{h}^{i} d x+\sum_{i=1}^{i_{o}} \tau \mathcal{A}\left(y_{h}^{i}, y_{h}^{i}\right)+\sum_{i=1}^{i_{o}} \tau \int_{\Omega} f\left(y_{h}^{i}\right) y_{h}^{i} d x+\sum_{i=1}^{i_{o}} \tau \int_{\Gamma} b\left[y_{h}^{i}\right]^{2} d s \\
=\sum_{i=1}^{i_{o}} \int_{t_{i-1}}^{t_{i}} \int_{\Omega} g(x, t) y_{h}^{i} d x d t
\end{gathered}
$$

which can also be written as follows:

$$
\begin{aligned}
& \sum_{i=1}^{i_{o}} \int_{\Omega}\left(y_{h}^{i}-y_{h}^{i-1}\right) y_{h}^{i} d x+\sum_{i=1}^{i_{o}} \tau \mathcal{A}\left(y_{h}^{i}, y_{h}^{i}\right)+\sum_{i=1}^{i_{o}} \tau \int_{\Omega}\left(f\left(y_{h}^{i}\right)-f(0)\right) y_{h}^{i} d x \\
& +\sum_{i=1}^{i_{o}} \tau \int_{\Omega} b\left[y_{h}^{i}\right]^{2} d s=\sum_{i=1}^{i_{o}} \int_{t_{i-1}}^{t_{i}} \int_{\Omega} g(x, t) y_{h}^{i} d x d t-\sum_{i=1}^{i_{o}} \tau \int_{\Omega} f(0) y_{h}^{i} d x .
\end{aligned}
$$

Taking (H1) into account, and using (3.3), we deduce from the above equality :

$$
\begin{gathered}
\sum_{i=1}^{i_{o}} \int_{\Omega}\left(y_{h}^{i}-y_{h}^{i-1}\right) y_{h}^{i} d x+m_{o} \sum_{i=1}^{i_{o}} \tau\left\|y_{h}^{i}\right\|_{H^{1}(\Omega)}^{2} \leq \\
K_{1}\left(\sum_{i=1}^{i_{o}} \int_{t_{i-1}}^{t_{i}}\|g(t)\|_{2, \Omega}\left\|y_{h}^{i}\right\|_{H^{1}(\Omega)} d t+\sum_{i=1}^{i_{o}} \tau|f(0)|\left\|y_{h}^{i}\right\|_{H^{1}(\Omega)}\right),
\end{gathered}
$$

where $K_{1}$ only depends on $\Omega$ and $d$. With the identity

$$
2 \sum_{i=1}^{i_{o}}\left(a_{i}-a_{i-1}\right) a_{i}=a_{i_{o}}^{2}-a_{o}^{2}+\sum_{i=1}^{i_{o}}\left(a_{i}-a_{i-1}\right)^{2},
$$


and the inequality $2 a b \leq \varepsilon a^{2}+\frac{b^{2}}{\varepsilon}$, we obtain:

$$
\begin{gathered}
2 m_{o} \sum_{i=1}^{i_{o}} \tau\left\|y_{h}^{i}\right\|_{H^{1}(\Omega)}^{2}+\left\|y_{h}^{i_{o}}\right\|_{2, \Omega}^{2}+\sum_{i=1}^{i_{o}}\left\|y_{h}^{i}-y_{h}^{i-1}\right\|_{2, \Omega}^{2} \leq \\
\left\|y_{h}^{o}\right\|_{2, \Omega}^{2}+m_{o} \sum_{i=1}^{i_{o}} \tau\left\|y_{h}^{i}\right\|_{H^{1}(\Omega)}^{2}+\frac{2 K_{1}}{m_{o}}\left[K_{2}\|g\|_{q, Q}^{2}+|f(0)|^{2}\right],
\end{gathered}
$$

where $K_{2}=K_{2}(\Omega, d, \sigma)$ is such that $\|\chi\|_{2, \Sigma}^{2} \leq K_{2}\|\chi\|_{\sigma, \Sigma}^{2}$ for all $\chi \in L^{\sigma}(\Sigma)$. Thanks to Proposition 3.1 and the bound $\|g\|_{q, Q} \leq M$, we finally obtain

$$
\sum_{i=1}^{i_{o}} \tau\left\|y_{h}^{i}\right\|_{H^{1}(\Omega)}^{2}+\left\|y_{h}^{i_{o}}\right\|_{2, \Omega}^{2}+\sum_{i=1}^{i_{o}}\left\|y_{h}^{i}-y_{h}^{i-1}\right\|_{2, \Omega}^{2} \leq K_{3}
$$

for any $i_{o} \in\{1, . ., N\}$, where $K_{3}$ only depends on $K_{1}, K_{2}, \Omega, M, m_{o}, C_{o}, \sigma, f(0),\left\|y_{o}\right\|_{H^{1}(\Omega)}$, and $h_{\Omega}$. This completes the proof.

ThEorem 3.2. Let $\delta=(h, \tau)$ be in $] 0, h_{\Omega}[\times] 0, T\left[\right.$. Let $g \in L^{q}(Q)$ be such that $\|g\|_{q, Q} \leq$ $M$, and let $y$ and $y_{\delta}$ be the solutions of (3.1) and (3.7)-(3.8) associated to $g$.

There exists a constant $C_{4}=C_{4}\left(\Omega, q, m_{o}, C_{o},\left\|y_{o}\right\|_{H^{1}}, M, h_{\Omega}\right)$ independent of $h, \tau, g$ such that:

$$
\left\|y_{\delta}-y\right\|_{L^{\infty}\left(0, T ; L^{2}(\Omega)\right)}^{2}+\sum_{i=1}^{N} \frac{1}{\tau}\left\|\int_{I_{i}}\left(y_{\delta}(t)-y(t)\right) d t\right\|_{H^{1}(\Omega)}^{2} d t \leq C_{4} \tau\left(1+\frac{h^{2}}{\tau^{2}}\right) .
$$

Proof - We set $\left.\left.e=y-y_{\delta}, y^{i}=y\left(t_{i}\right), y_{h}^{i}=y_{\delta}\left(t_{i}\right), I_{i}=\right] t_{i-1}, t_{i}\right]$ for $i \geq 1$.

We also set $\partial z^{i}=\frac{z^{i}-z^{i-1}}{\tau}, \bar{z}^{i}:=\frac{1}{\tau} \int_{I_{i}} z(t) d t$ for $i \geq 1$, and $\bar{z}^{o}=z_{o}$ for $i=0$. In particular we have $\bar{e}^{i}=\bar{y}^{i}-y_{h}^{i}$. In what follows, $C$ is a constant independent of $h, \tau$ which may depend on $M, m_{o}, C_{1}$. Setting $\varphi=\tau E_{h} \bar{e}^{i}$ in (3.7) yields :

$$
\tau\left\langle\partial y_{h}^{i}, E_{h} \bar{e}^{i}\right\rangle_{\Omega}+\tau\left(\mathcal{A}\left(y_{h}^{i}, E_{h} \bar{e}^{i}\right)+\left\langle f\left(y_{h}^{i}\right), E_{h} \bar{e}^{i}\right\rangle_{\Omega}+\left\langle b y_{h}^{i}, E_{h} \bar{e}^{i}\right\rangle_{\Gamma}\right)=\tau\left\langle\bar{g}^{i}, E_{h} \bar{e}^{i}\right\rangle_{\Omega} .
$$

On the other hand, for any $z \in H^{1}(\Omega)$, we have:

$$
\frac{d}{d t}\langle y(t), z\rangle_{\Omega}+\mathcal{A}(y(t), z)+\langle f(y(t)), z\rangle_{\Omega}+\langle b y(t), z\rangle_{\Gamma}=\langle g(t), z\rangle_{\Omega} .
$$

As $y \in W^{1,2, p}(Q)$, then $y^{i} \in H^{1}(\Omega)$ and we may choose $z=\bar{e}^{i}$ in the previous equality. Then summing over $I_{i}$ gives:

$$
\tau\left\langle\partial y^{i}, \bar{e}^{i}\right\rangle_{\Omega}+\tau\left(\mathcal{A}\left(\bar{y}^{i}, \bar{e}^{i}\right)+\left\langle\bar{f}^{i}, \bar{e}^{i}\right\rangle_{\Omega}+\left\langle b \bar{y}^{i}, \bar{e}^{i}\right\rangle_{\Gamma}\right)=\tau\left\langle\bar{g}^{i}, \bar{e}^{i}\right\rangle_{\Omega}
$$


We substract (3.9) from (3.10) and sum up from $i=1$ to $i=i_{o}\left(1 \leq i_{o} \leq N\right)$ :

$$
\begin{aligned}
(\mathrm{I})+(\mathrm{II}):= & \sum_{i=1}^{i_{o}} \tau\left\langle\partial\left(y^{i}-y_{h}^{i}\right), \bar{e}^{i}\right\rangle_{\Omega}+\sum_{i=1}^{i_{o}} \tau\left(a\left(\bar{y}^{i}, \bar{e}^{i}\right)-a\left(y_{h}^{i}, E_{h} \bar{e}^{i}\right)\right) \\
= & \sum_{i=1}^{i_{o}} \tau\left\langle\partial y_{h}^{i},\left(E_{h}-I\right) \bar{e}^{i}\right\rangle_{\Omega}+\sum_{i=1}^{i_{o}} \tau\left(C_{o}\left\langle\bar{y}^{i}, \bar{e}^{i}\right\rangle_{\Omega}-C_{o}\left\langle y_{h}^{i}, E_{h} \bar{e}^{i}\right\rangle_{\Omega}\right) \\
& +\sum_{i=1}^{i_{o}} \tau\left(\left\langle f\left(y_{h}^{i}\right), E_{h} \bar{e}^{i}\right\rangle_{\Omega}-\left\langle\bar{f}^{i}, \bar{e}^{i}\right\rangle_{\Omega}\right)+\sum_{i=1}^{i_{o}} \tau\left\langle\bar{g}^{i},\left(I-E_{h}\right) \bar{e}^{i}\right\rangle_{\Omega} \\
= & :(\mathrm{III})+(\mathrm{IV})+(\mathrm{V})+(\mathrm{VI}) .
\end{aligned}
$$

We now estimate each term of the above equality. First, we write (I) in the following form:

$$
\begin{aligned}
(\mathrm{I}) & =\sum_{i=1}^{i_{o}} \tau\left\langle\partial\left(y^{i}-y_{h}^{i}\right), \bar{e}^{i}\right\rangle_{\Omega} \\
& =\sum_{i=1}^{i_{o}} \tau\left\langle\partial \bar{e}^{i}, \bar{e}^{i}\right\rangle_{\Omega}+\sum_{i=1}^{i_{o}} \tau\left\langle\partial\left(y^{i}-\bar{y}^{i}\right), \bar{e}^{i}\right\rangle_{\Omega}=:(\mathrm{I})_{1}+(\mathrm{I})_{2} .
\end{aligned}
$$

Using the following identity (for any $F$ bilinear):

$$
2 \sum_{i=1}^{i_{o}} F\left(x_{i}-x_{i-1}, x_{i}\right)=F\left(x_{i_{o}}, x_{i_{o}}\right)-F\left(x_{o}, x_{o}\right)+\sum_{i=1}^{i_{o}} F\left(x_{i}-x_{i-1}, x_{i}-x_{i-1}\right),
$$

we obtain:

$$
(\mathrm{I})_{1}=\sum_{i=1}^{i_{o}}\left\langle\bar{e}^{i}-\bar{e}^{i-1}, \bar{e}^{i}\right\rangle_{\Omega}=\frac{1}{2}\left\|\bar{e}^{i_{\circ}}\right\|_{2, \Omega}^{2}-\frac{1}{2}\left\|\bar{e}^{0}\right\|_{2, \Omega}^{2}+\frac{1}{2} \sum_{i=1}^{i_{o}}\left\|\bar{e}^{i}-\bar{e}^{i-1}\right\|_{2, \Omega}^{2} .
$$

From Proposition 3.1, we get :

$$
(\mathrm{I})_{1} \geq C\left\|\bar{e}^{i_{o}}\right\|_{2, \Omega}^{2}-C h^{2}+C \sum_{i=1}^{i_{o}}\left\|\bar{e}^{i}-\bar{e}^{i-1}\right\|_{2, \Omega}^{2}
$$

Dealing with the estimate of $(\mathrm{I})_{2}$, we observe first that the following inequality holds:

$$
\left\|y(t)-\bar{y}^{i}\right\|_{2, \Omega} \leq \sqrt{\tau}\left\|\frac{\partial y}{\partial t}\right\|_{L^{2}\left(\Omega \times I_{i}\right)} \quad \forall i \in\{1, . ., N\} \text { and } t \in \overline{I_{i}}
$$

INRIA 
With $\bar{y}^{o}=y^{o}$ and (3.12), we have

$$
\begin{aligned}
\left|(\mathrm{I})_{2}\right| & =\left|\left\langle y^{i_{o}}-\bar{y}^{i_{o}}, \bar{e}^{i_{o}}\right\rangle_{\Omega}-\sum_{i=2}^{i_{o}}\left\langle y^{i-1}-\bar{y}^{i-1}, \bar{e}^{i}-\bar{e}^{i-1}\right\rangle_{\Omega}\right| \\
& \leq C \sqrt{\tau}\left\|\frac{\partial y}{\partial t}\right\|_{L^{2}\left(\Omega \times I_{i_{o}}\right)}\left\|\bar{e}^{i_{o}}\right\|_{2, \Omega}+C \sum_{i=2}^{i_{o}} \sqrt{\tau}\left\|\frac{\partial y}{\partial t}\right\|_{L^{2}\left(\Omega \times I_{i-1}\right)}\left\|\bar{e}^{i}-\bar{e}^{i-1}\right\|_{2, \Omega} \\
& \leq \varepsilon\left\|\bar{e}^{i_{o}}\right\|_{2, \Omega}^{2}+\varepsilon \sum_{i=1}^{i_{o}}\left\|\bar{e}^{i}-\bar{e}^{i-1}\right\|_{2, \Omega}^{2}+\frac{C}{\varepsilon} \tau\left\|\frac{\partial y}{\partial t}\right\|_{L^{2}\left(\Omega \times I_{i-1}\right)}^{2} \\
& \leq \varepsilon\left\|\bar{e}^{i_{o}}\right\|_{2, \Omega}^{2}+\varepsilon \sum_{i=1}^{i_{o}}\left\|\bar{e}^{i}-\bar{e}^{i-1}\right\|_{2, \Omega}^{2}+\frac{C}{\varepsilon} \tau\left\|\frac{\partial y}{\partial t}\right\|_{L^{2}(Q)}^{2}, \\
& \leq \varepsilon\left\|\bar{e}^{i_{o}}\right\|_{2, \Omega}^{2}+\varepsilon \sum_{i=1}^{i_{o}}\left\|\bar{e}^{i}-\bar{e}^{i-1}\right\|_{2, \Omega}^{2}+\frac{C}{\varepsilon} \tau
\end{aligned}
$$

where $\varepsilon>0$ will be chosen at the end of estimates. From the definition of $E_{h}$, it follows:

$$
\begin{aligned}
(\mathrm{II}) & =\sum_{i=1}^{i_{o}} \tau\left(a\left(\bar{y}^{i}, \bar{e}^{i}\right)-a\left(y_{h}^{i}, E_{h} \bar{e}^{i}\right)\right)=\sum_{i=1}^{i_{o}} \tau a\left(\bar{e}^{i}, E_{h} \bar{e}^{i}\right)=\sum_{i=1}^{i_{o}} \tau a\left(\bar{e}^{i}, \bar{e}^{i}\right) \\
& \geq C \sum_{i=1}^{i_{o}} \tau\left\|\bar{e}^{i}\right\|_{H^{1}(\Omega)}^{2} .
\end{aligned}
$$

The term (III) is bounded by means of ( $3.5 \mathrm{~b})$ and of Lemma 3.1 as follows:

$$
\begin{aligned}
|(\mathrm{III})| & =\left|\sum_{i=1}^{i_{o}} \tau\left\langle\partial y_{h}^{i},\left(E_{h}-I\right) \bar{e}^{i}\right\rangle_{\Omega}\right| \leq C_{2} \sum_{i=1}^{i_{o}}\left\|y_{h}^{i}-y_{h}^{i-1}\right\|_{2, \Omega} h\left\|\bar{e}^{i}\right\|_{H^{1}(\Omega)} \\
& \leq \varepsilon \tau \sum_{i=1}^{i_{o}}\left\|\bar{e}^{i}\right\|_{H^{1}(\Omega)}^{2}+\frac{C_{2}\left(C_{3}\right)^{2}}{\varepsilon} \frac{h^{2}}{\tau} \\
& \leq \varepsilon \tau \sum_{i=1}^{i_{o}}\left\|\bar{e}^{i}\right\|_{H^{1}(\Omega)}^{2}+\frac{C}{\varepsilon} \frac{h^{2}}{\tau} .
\end{aligned}
$$

$\mathrm{RR} \mathrm{n}^{\circ} 3940$ 
We bound (IV) using the same arguments as for (III):

$$
\begin{aligned}
\mid(\mathrm{IV})) \mid & \leq\left|C_{o} \sum_{i=1}^{i_{o}} \tau\left\langle\bar{y}^{i}-y_{h}^{i}, \bar{e}^{i}\right\rangle_{\Omega}\right|+\left|C_{o} \sum_{i=1}^{i_{o}} \tau\left\langle y_{h}^{i},\left(I-E_{h}\right) \bar{e}^{i}\right\rangle_{\Omega}\right| \\
& \leq\left|C_{o}\right| \sum_{i=1}^{i_{o}} \tau\left\|\bar{e}^{i}\right\|_{2, \Omega}^{2}+\left|C_{o}\right| \sum_{i=1}^{i_{o}} \tau\left\|y_{h}^{i}\right\|_{2, \Omega}\left\|\left(I-E_{h}\right) \bar{e}^{i}\right\|_{2, \Omega} \\
& \leq C \sum_{i=1}^{i_{o}} \tau\left\|\bar{e}^{i}\right\|_{2, \Omega}^{2}+C \sum_{i=1}^{i_{o}} \tau\left\|y_{h}^{i}\right\|_{H^{1}(\Omega)} h\left\|\bar{e}^{i}\right\|_{H^{1}(\Omega)} \\
& \leq C \sum_{i=1}^{i_{o}} \tau\left\|\bar{e}^{i}\right\|_{2, \Omega}^{2}+\varepsilon \sum_{i=1}^{i_{o}} \tau\left\|\bar{e}^{i}\right\|_{H^{1}(\Omega)}^{2}+\frac{C}{\varepsilon} h^{2} \sum_{i=1}^{i_{o}} \tau\left\|y_{h}^{i}\right\|_{H^{1}(\Omega)}^{2} \\
& \leq C \sum_{i=1}^{i_{o}} \tau\left\|\bar{e}^{i}\right\|_{2, \Omega}^{2}+\varepsilon \sum_{i=1}^{i_{o}} \tau\left\|\bar{e}^{i}\right\|_{H^{1}(\Omega)}^{2}+\frac{C}{\varepsilon} h^{2} .
\end{aligned}
$$

Now we bound the term involving non linearities. We rewrite $(\mathrm{V})$ in a suitable manner. In addition, we use the fact that $f$ is a lipschitz function and Lemma 2.1, which lead to:

$$
\begin{aligned}
|(\mathrm{V})| & \leq\left|\sum_{i=1}^{i_{o}} \tau\left\langle f\left(y_{h}^{i}\right)-\bar{f}^{i}, \bar{e}^{i}\right\rangle_{\Omega}\right|+\left|\sum_{i=1}^{i_{o}} \tau\left\langle f\left(y_{h}^{i}\right),\left(I-E_{h}\right) \bar{e}^{i}\right\rangle_{\Omega}\right| \\
& \leq \sum_{i=1}^{i_{o}}\left|\left\langle\int_{I_{i}}\left(f\left(y_{h}^{i}\right)-f(y(t))\right) d t, \bar{e}^{i}\right\rangle_{\Omega}\right|+\sum_{i=1}^{i_{o}} \tau\left|\left\langle f\left(y_{h}^{i}\right),\left(I-E_{h}\right) \bar{e}^{i}\right\rangle_{\Omega}\right| \\
& \leq C \sum_{i=1}^{i_{o}}\left\|\int_{I_{i}}\left|y_{h}^{i}-y(t)\right| d t\right\|_{2, \Omega}\left\|\bar{e}^{i}\right\|_{2, \Omega}+C \sum_{i=1}^{i_{o}} \tau\left(\left\|y_{h}^{i}\right\|_{2, \Omega}+|f(0)|\right)\left\|\left(E_{h}-I\right) \bar{e}^{i}\right\|_{2, \Omega} \\
& \leq \frac{\varepsilon}{2} \sum_{i=1}^{i_{o}} \tau\left\|\bar{e}^{i}\right\|_{2, \Omega}^{2}+\frac{C}{\varepsilon} \int_{0}^{t_{i_{o}}}\|e(t)\|_{2, \Omega}^{2} d t+C \sum_{i=1}^{i_{o}} \tau\left(\left\|y_{h}^{i}\right\|_{2, \Omega}+1\right) h\left\|\bar{e}^{i}\right\|_{H^{1}(\Omega)} \\
& \leq \frac{\varepsilon}{2} \sum_{i=1}^{i_{o}} \tau\left\|\bar{e}^{i}\right\|_{H^{1}(\Omega)}^{2}+\frac{C}{\varepsilon} \int_{0}^{t_{i o}}\|e(t)\|_{2, \Omega}^{2}+\frac{C\left(C_{2}+1\right)}{\varepsilon} h^{2}+\frac{\varepsilon}{2} \sum_{i=1}^{i_{o}} \tau\left\|\bar{e}^{i}\right\|_{H^{1}(\Omega)}^{2} \\
& \leq \frac{C}{\varepsilon} \int_{0}^{t_{i_{o}}}\|e(t)\|_{2, \Omega}^{2} d t+\frac{C}{\varepsilon} h^{2}+\varepsilon \sum_{i=1}^{i_{o}} \tau\left\|\bar{e}^{i}\right\|_{H^{1}(\Omega)}^{2} .
\end{aligned}
$$


Since $\|g\|_{q, Q} \leq M$, we have:

$$
\begin{aligned}
|(\mathrm{VI})| & =\left|\sum_{i=1}^{i_{o}} \tau\left\langle\bar{g}^{i},\left(E_{h}-I\right) \bar{e}^{i}\right\rangle_{\Omega}\right| \leq C \sum_{i=1}^{i_{o}}\left(\int_{I_{i}}\|g(t)\|_{2, \Omega}^{2} d t\right)^{1 / 2} h \sqrt{\tau}\left\|\bar{e}^{i}\right\|_{H^{1}(\Omega)} \\
& \leq \frac{C}{\varepsilon} h^{2} \sum_{i=1}^{i_{o}}\|g\|_{2, I_{i} \times \Omega}^{2}+\varepsilon \sum_{i=1}^{i_{o}} \tau\left\|\bar{e}^{i}\right\|_{H^{1}(\Omega)}^{2} \leq \frac{C}{\varepsilon} h^{2}+\varepsilon \sum_{i=1}^{i_{o}} \tau\left\|\bar{e}^{i}\right\|_{H^{1}(\Omega)}^{2} .
\end{aligned}
$$

To summarize, we have proved the following estimate:

$$
\begin{aligned}
& \left\|\bar{e}^{i_{o}}\right\|_{2, \Omega}^{2}+\sum_{i=1}^{i_{o}}\left\|\bar{e}^{i}-\bar{e}^{i-1}\right\|_{2, \Omega}^{2}+\sum_{i=1}^{i_{o}} \tau\left\|\bar{e}^{i}\right\|_{H^{1}(\Omega)}^{2} \leq \\
& \frac{C}{\varepsilon}\left(\tau+\frac{h^{2}}{\tau}+h^{2}\right)+\frac{C}{\varepsilon} \int_{0}^{t_{i_{o}}}\|e(t)\|_{2, \Omega}^{2} d t+C \sum_{i=1}^{i_{o}} \tau\left\|\bar{e}^{i}\right\|_{2, \Omega}^{2} \\
& +C \varepsilon\left\|\bar{e}^{i_{o}}\right\|_{2, \Omega}^{2}+C \varepsilon \sum_{i=1}^{i_{o}}\left\|\bar{e}^{i}-\bar{e}^{i-1}\right\|_{2, \Omega}^{2}+4 C \varepsilon \sum_{i=1}^{i_{o}} \tau\left\|\bar{e}^{i}\right\|_{H^{1}(\Omega)}^{2} .
\end{aligned}
$$

Now we set $C \varepsilon=\frac{1}{6}$ so that the last three terms of the right-hand side in inequality (3.13) are controlled by the terms of the left-hand side of (3.13). Thus, for any $i_{o} \in\{1, \ldots, N\}$, we have:

$$
\left\|\bar{e}^{i_{o}}\right\|_{2, \Omega}^{2}+\sum_{i=1}^{i_{o}} \tau\left\|\bar{e}^{i}\right\|_{H^{1}(\Omega)}^{2} \leq C\left(\tau+\frac{h^{2}}{\tau}+h^{2}\right)+C \int_{0}^{t_{i_{o}}}\|e(t)\|_{2, \Omega}^{2} d t+C \sum_{i=1}^{i_{o}} \tau\left\|\bar{e}^{i}\right\|_{2, \Omega}^{2} .
$$

Let us observe that for $i \in\{1, \ldots, N\}$ and $\left.t \in] t_{i-1}, t_{i}\right]$, we have:

$$
\begin{aligned}
\|e(t)\|_{2, \Omega}^{2} & \leq 2\left\|y(t)-\bar{y}^{i}\right\|_{2, \Omega}^{2}+2\left\|\bar{y}^{i}-y_{h}^{i}\right\|_{2, \Omega}^{2} \\
& \leq 2 \tau\left\|\frac{\partial y}{\partial t}\right\|_{L^{2}(Q)}^{2}+2\left\|\bar{e}^{i}\right\|_{2, \Omega}^{2} \\
& \leq 2\left(C_{2} \tau+\left\|\bar{e}^{i}\right\|_{2, \Omega}^{2}\right) .
\end{aligned}
$$

This observation with (3.14) yields:

$$
\left\|\bar{e}^{i_{o}}\right\|_{2, \Omega}^{2}+\sum_{i=1}^{i_{o}} \tau\left\|\bar{e}^{i}\right\|_{H^{1}(\Omega)}^{2} \leq C\left(\tau+\frac{h^{2}}{\tau}+h^{2}\right)+C \sum_{i=1}^{i_{o}} \tau\left\|\bar{e}^{i}\right\|_{2, \Omega}^{2} .
$$

Using a discrete Gronwall inequality (see Lemma 3.2), we obtain:

$$
\left\|\bar{e}^{i_{o}}\right\|_{2, \Omega}^{2}+\sum_{i=1}^{i_{o}} \tau\left\|\bar{e}^{i}\right\|_{H^{1}(\Omega)}^{2} \leq C\left(\tau+\frac{h^{2}}{\tau}+h^{2}\right)
$$


From (3.15), we have $\sup _{t \in] 0,1]}\|\bar{e}(t)\|_{2, \Omega}^{2} \leq C\left(\max _{1 \leq i \leq N}\left\|\bar{e}^{i}\right\|_{2, \Omega}^{2}+\tau\right)$. This estimate together with (3.17) gives:

$$
\sup _{t \in] 0,1]}\|e(t)\|_{2, \Omega}^{2}+\sum_{i=1}^{i_{o}} \tau\left\|\bar{e}^{i}\right\|_{H^{1}(\Omega)}^{2} \leq C\left(\tau+\frac{h^{2}}{\tau}+h^{2}\right) .
$$

This completes the proof.

Lemma 3.2. ( Discrete Gronwall inequality).

Let $\left(a_{n}\right)_{n}$ and $\left(b_{n}\right)_{n}$ be two sequences of positive numbers, let $\left(c_{n}\right)_{n}$ be an increasing sequence of positive numbers, and let $\tau>0$ such that

$$
a_{n}+b_{n} \leq c_{n}+\sum_{k=0}^{n-1} \tau a_{k} \quad \forall n \geq 1,
$$

with $a_{o}+b_{o} \leq c_{o}$. Then, the following inequality holds:

$$
a_{n}+b_{n} \leq c_{n} \exp (\tau n) \quad \forall n .
$$

Proof - We can find this version of Gronwall's inequality in [21] p. 43 (see also [17]).

\section{Approximation of the optimal control problem.}

4.1. Relaxation of the constraints. In [4], in order to study the approximation of optimal control problems governed by elliptic equations, the state equation is discretized with a finite element method and the state constraint is replaced by a finite number of constraints at the nodes of the discretization. Notice that error estimates for discretizations of elliptic equations (in dimension 3 or less than 3) can be obtained for the norm of $\mathcal{C}(\bar{\Omega})$, which allows to obtain the convergence of the discretization scheme analyzed in [4] and [5].

In the case of parabolic equations, we are only able to establish error estimates for the $L^{\infty}\left(L^{2}\right)$-norm, and thus we cannot deal with the state constraints as in [4], [5]. Therefore, we must give an integral form for these pointwise contraints. In addition, we must ensure that the discretized problem has a solution, i.e. the "discretized" feasible domain is non empty. A good candidate to be an element of this discretized feasible domain is of course the discrete approximation of the solution to the continuous problem (which satisfies the continuous constraints). Unfortunately, the constaints are not necessarly satisfied a priori for the discretized solution even if the discretization step $\delta$ is small. Therefore, we are obliged to relax these constraints with respect to some $\varepsilon$ small enough (see the proof of proposition 4.3 below).

Thus, we consider a (continuous) penalized problem $\left(\mathcal{P}^{\varepsilon}\right)$ defined for $\varepsilon>0$ as follows:

$$
\begin{aligned}
& \inf \left\{J(y, v, \theta) \mid(y, v, \theta) \in \mathcal{C}(\bar{Q}) \times V_{a d},(y, v, \theta) \text { satisfies }(2.2 a)\right. \text { and } \\
& \left.\int_{Q}\left((y-\psi)^{-}\right)^{2} d x d t \leq \varepsilon, \int_{Q}\left(F^{+}(x, t, y(x, t))\right)^{2} d x d t \leq \varepsilon,\left(\int_{Q}(y-\psi) \theta d x d t\right)^{2} \leq \varepsilon\right\} .
\end{aligned}
$$


Here $F^{+}$denotes the nonnegative part of $F$ (and $y^{-}$the negative part of $y$ ). Since $V_{a d}$ is bounded in $L^{q}(Q) \times L^{q}(Q)$ and the state equation is linear with respect to the control variables, the problem $(\widetilde{\mathcal{P}})$ is weakly stable to the right in the following sense.

Proposition 4.1. We have $\lim _{\varepsilon \rightarrow 0}\left(\inf \left(\mathcal{P}^{\varepsilon}\right)\right)=\inf (\widetilde{\mathcal{P}})=\inf (\mathcal{P})$.

Proof - Step 1: We fix $\varepsilon>0$ and we prove that the problem $\left(\mathcal{P}^{\varepsilon}\right)$ has at least one solution $\left(y_{\varepsilon}, v_{\varepsilon}, \theta_{\varepsilon}\right)$. It's clear that the feasible domain of $\left(\mathcal{P}^{\varepsilon}\right)$ is non empty (any solution of $(\widetilde{\mathcal{P}})$ is feasible for $\left.\left(\mathcal{P}^{\varepsilon}\right)\right)$. Let $\left(y_{n}, v_{n}, \theta_{n}\right)_{n \geq 1}$ be a minimizing sequence of $\left(\mathcal{P}^{\varepsilon}\right)$. The sequence $\left(v_{n}, \theta_{n}\right)_{n}$ is included in $V_{a d}$ and is bounded in $L^{q} \times L^{q}(Q)$; therefore there exists a subsequence, still denoted by $\left(v_{n}, \theta_{n}\right)_{n}$, converging to some $\left(v_{\varepsilon}, \theta_{\varepsilon}\right)$ in $L^{q} \times L^{q}(Q)$. Since $V_{a d}$ is convex closed, it's also weakly closed and then $\left(v_{\varepsilon}, \theta_{\varepsilon}\right)$ belongs to $V_{a d}$. In addition, theorem 1.2 gives the strong convergence in $\mathcal{C}(\bar{Q})$ of $\left(y_{n}\right)_{n}$ to the solution $y_{\varepsilon}$ of $(2.2 \mathrm{a})$ corresponding to $\left(v_{\varepsilon}, \theta_{\varepsilon}\right)$. On the other hand, since $\left(y_{n}, v_{n}, \theta_{n}\right)$ is feasible for $\left(\mathcal{P}^{\varepsilon}\right)$, we have:

$$
\int_{Q}\left(\left(y_{n}-\psi\right)^{-}\right)^{2} d x d t \leq \varepsilon, \int_{Q}\left(F^{+}\left(x, t, y_{n}(x, t)\right)\right)^{2} d x d t \leq \varepsilon,\left(\int_{Q}\left(y_{n}-\psi\right) \theta_{n} d x d t\right)^{2} \leq \varepsilon,
$$

for every $n \geq 1$. By passing to the limit, when $n \rightarrow \infty$, we deduce that

$$
\int_{Q}\left(\left(y_{\varepsilon}-\psi\right)^{-}\right)^{2} d x d t \leq \varepsilon, \int_{Q}\left(F^{+}\left(x, t, y_{\varepsilon}(x, t)\right)\right)^{2} d x d t \leq \varepsilon,\left(\int_{Q}\left(y_{\varepsilon}-\psi\right) \theta_{\varepsilon} d x d t\right)^{2} \leq \varepsilon
$$

and then $\left(y_{\varepsilon}, v_{\varepsilon}, \theta_{\varepsilon}\right)$ is feasible for the problem $\left(\mathcal{P}^{\varepsilon}\right)$. By the continuity of $J$ with respect to $y$ and the convexity and continuity assumption with respect to $v$ (see (H5)-(H6)), we conclude:

$$
\operatorname{Inf}\left(\mathcal{P}^{\varepsilon}\right) \leq J\left(y_{\varepsilon}, v_{\varepsilon}\right) \leq \liminf _{n} J\left(y_{n}, v_{n}\right)=\operatorname{Inf}\left(\mathcal{P}^{\varepsilon}\right) .
$$

Therefore, $\left(y_{\varepsilon}, v_{\varepsilon}, \theta_{\varepsilon}\right)$ is a solution of $\left(\mathcal{P}^{\varepsilon}\right)$.

Step 2: Now we study the asymptotic behavior of $\left(y_{\varepsilon}, v_{\varepsilon}, \theta_{\varepsilon}\right)_{\varepsilon}$. Again, since $V_{a d}$ is bounded in $L^{q}(Q) \times L^{q}(Q)$, there exists a sequence $\left\{\varepsilon_{j}\right\}_{j=1}^{\infty}$ and $(\bar{v}, \bar{\theta}) \in V_{a d}$ such that $\varepsilon_{j} \rightarrow 0, v_{\varepsilon_{j}} \rightarrow \bar{v}$ weakly in $L^{q}(Q)$, and $\theta_{\varepsilon_{j}} \rightarrow \bar{\theta}$ in $L^{q}(Q)$ when $j \rightarrow \infty$. If we denote by $y_{\varepsilon_{j}}$ and $\bar{y}$ the states associated respectively to $\left(v_{\varepsilon_{j}}, \theta_{\varepsilon_{j}}\right)$ and to $(\bar{v}, \bar{\theta})$, Theorem 1.2 yields that $y_{\varepsilon_{j}} \rightarrow \bar{y}$ uniformly in $\bar{Q}$. By the same arguments as in Step 1 , we can check that $(\bar{y}, \bar{v}, \bar{\theta})$ is feasible for $(\widetilde{\mathcal{P}})$. Using again the convexity of $\tilde{J}$ with respect to the second variable and the feasibility of $(\bar{y}, \bar{v}, \bar{\theta})$ for $(\widetilde{\mathcal{P}})$, we get

$$
\inf (\widetilde{\mathcal{P}}) \leq J(\bar{y}, \bar{v}) \leq \liminf _{j \rightarrow \infty} J\left(y_{\varepsilon_{j}}, v_{\varepsilon_{j}}\right)=\lim _{j \rightarrow \infty} \inf \left(\mathcal{P}^{\varepsilon_{j}}\right) \leq \inf (\widetilde{\mathcal{P}}),
$$

which concludes the proof.

REMARK 4.1. The stability considered in the above proposition has been already introduced by many authors (see [4,5] for example) in order to study the approximation of optimal control problems governed by elliptic equations. 
REMARK 4.2. We may remark that the relaxation of the bilinear integral constraint is needed anyway if a related Lagrange multiplier is expected [2]. Indeed, this constraint is too stressing, so that usual regularity conditions (see [23] for example) cannot be satisfied. Therefore, it is not possible to ensure the existence of a Lagrange multiplier associated to this constraint.

4.2. The discretized problem. We recall that for any $h$ and $\tau$ (space and time discretization steps), we set $\delta=(h, \tau)$ and we consider the space $\mathcal{V}_{\delta}$ defined by:

$$
\mathcal{V}_{\delta}=\left\{v_{\delta} \in L^{q}(Q)\left|v_{\delta}\right|_{\left.K \times] t_{i-1}, t_{i}\right]} \text { is constant for any } K \in \widetilde{\mathcal{F}}_{h} \text { and any } i=1, \ldots, N\right\} \text {. }
$$

Any function $v_{\delta}$ of $\mathcal{V}_{\delta}$ may be written as

$$
v_{\delta}(x, t)=\sum_{i=1}^{N} \sum_{j=1}^{N_{e}} V_{j}^{i} \chi_{i}(t) \chi_{K_{j}}(x)
$$

where $\chi_{K_{j}}$ is the characteristic function of $K_{j}$ and $V_{j}^{i} \in \mathbb{R}$. Any function $v$ of $L^{q}(Q)$ can be approximated by $v_{\delta}$ as in (4.2) where

$$
V_{j}^{i}=\frac{1}{\left|Q_{i j}\right|} \int_{Q_{i j}} v(x, t) d x d t \quad \text { for any } i=1, \ldots, N, j=1, \ldots, N_{e},
$$

where $\left.\left.Q_{i j}=K_{j} \times\right] t_{i-1}, t_{i}\right]$ and $\left|Q_{i j}\right|$ is the measure of $Q_{i j}$.

Proposition 4.2. Assume $v \in L^{q}(Q)$ and $v_{\delta} \in \mathcal{V}_{\delta}$ is defined as above. Then

$$
\lim _{\delta \rightarrow 0}\left\|v_{\delta}-v\right\|_{q, Q}=0 \text {. }
$$

Proof - Let $(x, t) \in Q$ where $v(x, t)$ makes sense and $Q_{\delta_{k}}=Q_{i_{\delta} j_{\delta}}$ a sequence of discretized cells which tends to $\{(x, t)\}$ as $\delta \rightarrow 0$. Extending $v$ by 0 outside $Q$ we have $v \in L^{1}\left(\mathbb{R}^{d+1}\right)$ and a classical result (see [16] for example) yields that

$$
\frac{1}{\left|Q_{\delta_{k}}\right|} \int_{Q_{\delta_{k}}} v(\xi, s) d \xi d s \rightarrow v(x, t) \text { as } \delta \rightarrow 0 .
$$

Therefore $v_{\delta_{k}}$ converges to $v$ almost everywhere, and $v_{\delta_{k}}^{q}$ converges to $v^{q}$ as well. In addition, with Hölder inequality we get

$$
\left|V_{j}^{i}\right|^{q} \leq \frac{1}{\left|Q_{i j}\right|} \int_{Q_{i j}}|v(x, t)|^{q} d x d t
$$

and we obtain

$$
\left\|v_{\delta}\right\|_{q, Q}^{q}=\sum_{i=1}^{N} \sum_{j=1}^{N_{e}}\left|Q_{i j}\right|\left|V_{j}^{i}\right|^{q} \leq \sum_{i=1}^{N} \sum_{j=1}^{N_{e}} \int_{Q_{i j}}|v(x, t)|^{q} d x d t \leq\|v\|_{q, Q}^{q} .
$$


We conclude with the Lebesgue dominated convergence theorem.

The end of the proposition follows from the uniqueness of the weak limit.

Similarly, we recall that, for $\delta=(h, \tau)$, the discretized solution of the state equation is defined as

$$
\left\{\begin{array}{l}
\left.\left.y_{\delta}(x, t)=\sum_{i=1}^{N} \chi_{i}(t) y_{h}^{i}(x)=\sum_{i=1}^{N} \sum_{j=1}^{N_{e}} Y_{j}^{i} \chi_{i}(t) \varphi_{j}(x) \quad \text { in } \Omega \times\right] 0, T\right] \\
y_{\delta}(\cdot, 0)=E_{h} y_{o}
\end{array}\right.
$$

We call $\mathcal{Y}_{\delta}$ the finite dimensional space which basis is $\left(\chi_{i} \varphi_{j}\right)_{i=1, \ldots, N, j=1, \ldots, N_{e}}$. This space dimension is $N_{t o t}=N * N_{e}$. In the sequel we shall set

$$
\begin{aligned}
& V^{i}=\left(V_{j}^{i}\right)_{j=1, \ldots, N_{e}} \in \mathbb{R}^{N_{e}}, V=\left(V^{i}\right)_{i=1, \ldots, N} \in \mathbb{R}^{N_{t o t}}, \\
& Y^{i}=\left(Y_{j}^{i}\right)_{j=1, \ldots, N_{e}} \in \mathbb{R}^{N_{e}}, Y=\left(Y^{i}\right)_{i=1, \ldots, N} \in \mathbb{R}^{N_{\text {tot }}} .
\end{aligned}
$$

We define now the well known mass and stiffness matrices:

$$
[M]=\left[\int_{\Omega} \varphi_{i}(x) \varphi_{j}(x) d x\right]_{1 \leq i, j \leq N_{e}} \text { and } \quad[R]=\left[\int_{\Omega} D \varphi_{i}(x) D \varphi_{j}(x) d x\right]_{1 \leq i, j \leq N_{e}} .
$$

With these notations, we have

$$
\left\|y_{h}^{i}\right\|_{H^{1}(\Omega)}^{2}=\left(Y^{i}\right)^{\top}([M]+[R]) Y^{i},
$$

where $Z^{\top}$ denotes the transposed vector of $Z$. We recall that $[M]$ and $[R]$ are symmetric, definite positive. Let us detail the discretized equation (3.7). It is equivalent to

$$
\left\{\begin{array}{l}
\text { For } i=1, \ldots, N \text { and } j=1, \ldots, N_{e} \\
\frac{1}{\tau} \sum_{k=1}^{N_{e}}\left(\int_{\Omega} \varphi_{k} \varphi_{j} d x\right)\left(Y_{k}^{i}-Y_{k}^{i-1}\right)+\sum_{k=1}^{N_{e}} \mathcal{A}\left(\varphi_{k}, \varphi_{j}\right) Y_{k}^{i} \\
+\int_{\Omega} f\left(y_{h}^{i}\right) \varphi_{j} d x+\sum_{k=1}^{N_{e}}\left(\int_{\Gamma} b \varphi_{k} \varphi_{j} d s\right) Y_{k}^{i}=\frac{1}{\tau} \int_{t_{i-1}}^{t_{i}} \int_{\Omega} g(\cdot, t) \varphi_{j} d x d t \\
y_{h}^{o}:=E_{h} y_{o} .
\end{array}\right.
$$

Let us set

$$
[A]=\left[\mathcal{A}\left(\varphi_{k}, \varphi_{j}\right)\right]_{1 \leq k, j \leq N_{e}} \quad \text { and } \quad[B]=\left[\int_{\Gamma} b \varphi_{k} \varphi_{j} d s\right]_{1 \leq k, j \leq N_{e}},
$$

and for every $i=1, \ldots, N$

$\Phi\left(Y^{i}\right)=\left(\int_{\Omega} f\left(y_{h}^{i}(x)\right) \varphi_{j}(x) d x\right)_{j=1, \ldots, N_{e}}, H_{i}(g)=\left(\int_{t_{i-1}}^{t_{i}} \int_{\Omega} g(x, t) \varphi_{j}(x) d x d t\right)_{j=1, \ldots, N_{e}}$. 
Then the above relation is equivalent to

$$
\left\{\begin{array}{l}
\text { For } i=1, \ldots, N \\
([M]+\tau[A]+\tau[B]) Y^{i}+\tau \Phi\left(Y^{i}\right)=[M] Y^{i-1}+H_{i}(g), \\
y_{h}^{o}:=E_{h} y_{o} .
\end{array}\right.
$$

Now we may define the discretized problem corresponding to $\left(\mathcal{P}^{\varepsilon}\right)$. For any $\left(v_{\delta}, \theta_{\delta}\right)$ in $\mathcal{V}_{\delta} \times \mathcal{V}_{\delta}$ we denote by $y_{\delta}\left(v_{\delta}, \theta_{\delta}\right)$ the solution of (4.3)-(4.5) associated to $g=v_{\delta}+\theta_{\delta}$. We set

$$
V_{a d, \delta}=V_{a d} \cap\left(\mathcal{V}_{\delta} \times \mathcal{V}_{\delta}\right)
$$

and

$$
\psi_{\delta}(x, t)=\sum_{i=1}^{N} \sum_{j=1}^{N_{e}} \Psi_{j}^{i} \chi_{i}(t) \varphi_{j}(x) \text { where } \Psi_{j}^{i}=\psi\left(x_{j}, t_{i}\right) .
$$

For any $\varepsilon>0$, we define

$$
\left\{\begin{array}{l}
\operatorname{minimize} \quad J\left(y_{\delta}, v_{\delta}, \theta_{\delta}\right) \\
\text { with }\left(v_{\delta}, \theta_{\delta}\right) \in V_{a d, \delta} \text { and } y_{\delta}=y_{\delta}\left(v_{\delta}, \theta_{\delta}\right) \\
\int_{Q}\left(\left(y_{\delta}-\psi_{\delta}\right)^{-}\right)^{2} d x d t \leq \varepsilon, \int_{Q}\left(F^{+}\left(x, t, y_{\delta}\right)\right)^{2} d x d t \leq \varepsilon \\
\left(\int_{Q} \theta_{\delta}\left(y_{\delta}-\psi_{\delta}\right) d x d t\right)^{2} \leq \varepsilon
\end{array}\right.
$$

We may now ascertain that the discretized problem has a solution :

Proposition 4.3. Given $\varepsilon>0$, there exists $\delta^{*}=\left(h^{*}, \tau^{*}\right)$ such that for all $\delta=(h, \tau) \in$ $\left.\left.\left[0, h^{*}\right] \times\right] 0, \tau^{*}\right]$ the problem $\left(\mathcal{P}_{\delta}^{\varepsilon}\right)$ has a solution.

Proof - Let us fix $\varepsilon>0$ and let us prove that there exists $\delta^{*}=\left(h^{*}, \tau^{*}\right)$ such that for all $\left.\left.\left.\delta \in] 0, h^{*}\right] \times\right] 0, \tau^{*}\right], \frac{h}{\tau}=O(1)$, the feasible domain $\mathcal{D}_{\delta}^{\varepsilon}$ of $\left(\mathcal{P}_{\delta}^{\varepsilon}\right)$ is non empty.

Let $(\bar{y}, \bar{v}, \bar{\theta})$ be a solution of $(\widetilde{\mathcal{P}})$ and let $\left(v_{\delta}, \theta_{\delta}\right)$ be the discrete approximation of $(\bar{v}, \bar{\theta})$ which is known to belong to $V_{a d, \delta}$ and converge to $(\bar{v}, \bar{\theta})$ in $L^{q}(Q) \times L^{q}(Q)$ (Proposition 4.2). Let $y_{\delta}=y_{\delta}\left(v_{\delta}, \theta_{\delta}\right)$ be the solution of (4.3)-(4.5) associated to $g=v_{\delta}+\theta_{\delta}$, and $y\left(v_{\delta}, \theta_{\delta}\right)$ the solution of $(2.2 \mathrm{a})$ associated to $\left(v_{\delta}, \theta_{\delta}\right)$. Since we have

$$
\left\|y_{\delta}-\bar{y}\right\|_{L^{\infty}\left(0, T ; L^{2}(\Omega)\right)}^{2} \leq\left\|y_{\delta}-y\left(v_{\delta}, \theta_{\delta}\right)\right\|_{L^{\infty}\left(0, T ; L^{2}(\Omega)\right)}^{2}+\left\|y\left(v_{\delta}, \theta_{\delta}\right)-\bar{y}\right\|_{L^{\infty}\left(0, T ; L^{2}(\Omega)\right)}^{2},
$$

Theorem 3.2 yields

$$
\left\|y_{\delta}-\bar{y}\right\|_{L^{\infty}\left(0, T ; L^{2}(\Omega)\right)}^{2} \leq C_{4} \tau\left(1+\frac{h^{2}}{\tau^{2}}\right)+\left\|y\left(v_{\delta}, \theta_{\delta}\right)-\bar{y}\right\|_{L^{\infty}\left(0, T ; L^{2}(\Omega)\right)}^{2}
$$


and by theorem 1.2 we conclude that $\left\|y_{\delta}-\bar{y}\right\|_{L^{\infty}\left(L^{2}\right)} \rightarrow 0$ as $\delta=(h, \tau) \rightarrow 0$ with $\frac{h}{\tau}=O(1)$. Since $\bar{y}$ satisfies the constraints:

$$
\int_{Q}\left((\bar{y}-\psi)^{-}\right)^{2} d x d t=0, \quad \int_{Q}\left(F^{+}(x, t, \bar{y})\right)^{2} d x d t=0,\left(\int_{Q} \bar{\theta}(\bar{y}-\psi) d x d t\right)^{2}=0,
$$

the function $y_{\delta}$ satisfies

$$
\int_{Q}\left(\left(y_{\delta}-\psi_{\delta}\right)^{-}\right)^{2} d x d t \leq \varepsilon, \int_{Q}\left(F^{+}\left(x, t, y_{\delta}\right)\right)^{2} d x d t \leq \varepsilon,\left(\int_{Q} \theta_{\delta}\left(y_{\delta}-\psi_{\delta}\right) d x d t\right)^{2} \leq \varepsilon,
$$

as soon as $\delta=(h, \tau)$ is small enough, say $\delta \leq \delta^{*}$ where $\delta^{*}$ depends on $\varepsilon$. Therefore, $\mathcal{D}_{\delta}^{\varepsilon}$ is non empty for such $\delta$.

Since $\mathcal{D}_{\delta}^{\varepsilon}$ is closed and nonempty and thanks to (H4)-(H6), the end of the proof is classical.

We end this section with a convergence result of the solution to the discretized problem $\left(\mathcal{P}_{\delta}^{\varepsilon}\right)$ to the solution of the continuous problem $\left(\mathcal{P}^{\varepsilon}\right)$.

Theorem 4.1. We fix $\varepsilon>0$. If $\left(v_{\delta}^{\varepsilon}, \theta_{\delta}^{\varepsilon}\right)_{\delta \leq \delta^{*}}$ denotes a solution to $\left(\mathcal{P}_{\delta}^{\varepsilon}\right)$, one can extract a subsequence weakly converging towards $\left(v_{\varepsilon}, \theta_{\varepsilon}\right)$ in $L^{q}(Q) \times L^{q}(Q)$, where $\left(v_{\varepsilon}, \theta_{\varepsilon}\right)$ is a solution to $\left(\mathcal{P}^{\varepsilon}\right)$. In addition we get

$$
\lim _{\delta \rightarrow 0} \inf \left(\mathcal{P}_{\delta}^{\varepsilon}\right)=\inf \left(\mathcal{P}^{\varepsilon}\right)
$$

Proof - The sequence $\left(v_{\delta}^{\varepsilon}, \theta_{\delta}^{\varepsilon}\right)_{\delta}$ belongs to $V_{a d}$ and is bounded in $L^{q}(Q) \times L^{q}(Q)$ (uniformly with respect to $\delta)$; therefore, there exists a subsequence (still denoted $\left.\left(v_{\delta}^{\varepsilon}, \theta_{\delta}^{\varepsilon}\right)_{\delta}\right)$ and $\left(v_{\varepsilon}, \theta_{\varepsilon}\right) \in$ $V_{a d}$, such that $\left(v_{\delta}^{\varepsilon}, \theta_{\delta}^{\varepsilon}\right)_{\delta}$ weakly converges towards $\left(v_{\varepsilon}, \theta_{\varepsilon}\right)$ in $L^{q}(Q) \times L^{q}(Q)$ as $\delta \rightarrow 0$.

Let $y_{\delta}^{\varepsilon}$ be the solution to (4.3)-(4.5) associated to $v_{\delta}^{\varepsilon}+\theta_{\delta}^{\varepsilon}$, and $y_{\varepsilon}$ be the solution of (2.2a) associated to $\left(v_{\varepsilon}, \theta_{\varepsilon}\right)$. We know with Theorem 3.2, that

$$
\lim _{\delta \rightarrow 0}\left\|y_{\delta}^{\varepsilon}-y\left(v_{\delta}^{\varepsilon}, \theta_{\delta}^{\varepsilon}\right)\right\|_{2, Q}=0
$$

where $y\left(v_{\delta}^{\varepsilon}, \theta_{\delta}^{\varepsilon}\right)$ is the solution to the (continuous) state-equation (2.2a) associated to $\left(v_{\delta}^{\varepsilon}, \theta_{\delta}^{\varepsilon}\right)$. In addition,

$$
\lim _{\delta \rightarrow 0}\left\|y\left(v_{\delta}^{\varepsilon}, \theta_{\delta}^{\varepsilon}\right)-y_{\varepsilon}\right\|_{2, Q}=0
$$

using the compactness result of Theorem 1.2 .

Hence $y_{\delta}^{\varepsilon}$ strongly converges towards $y_{\varepsilon}$ in $L^{2}(Q)$ (and even in $L^{\infty}\left(0, T ; L^{2}(\Omega)\right)$ ). Since $\left(y_{\delta}^{\varepsilon}, v_{\delta}^{\varepsilon}, \theta_{\delta}^{\varepsilon}\right)$ satisfies the constraints of the problem $\left(\mathcal{P}_{\delta}^{\varepsilon}\right)$, by passing to the limit when $\delta$ tends to 0 and taking the lipschitz continuity of $F$ and the continuity of $\psi$ into account (see (H4)-(H5)), we obtain

$$
\int_{Q}\left(F^{+}\left(x, t, y_{\varepsilon}\right)\right)^{2} d x d t \leq \varepsilon, \quad \int_{Q}\left(\left(y_{\varepsilon}-\psi\right)^{-}\right)^{2} d x d t \leq \varepsilon,
$$


and

$$
\left(\int_{Q}\left(y_{\varepsilon}-\psi\right) \theta_{\varepsilon} d x d t\right)^{2} \leq \varepsilon
$$

Therefore $\left(y_{\varepsilon}, v_{\varepsilon}, \theta_{\varepsilon}\right)$ is feasible for $\left(\mathcal{P}^{\varepsilon}\right)$. The end of the proof is a consequence of the semi-continuity on $L^{q}(Q)$-weak of the functional $J$ to minimize.

REMARK 4.3. The relaxation of the constraints via $\varepsilon$ is imposed by the necessity to ensure the non vacuity of the discretized feasible domain. Of course, this is unuseful if we are able to ascertain that $\mathcal{D}_{\delta}^{o}$ is non empty (i.e. the discretized feasible domain corresponding to " $\varepsilon=0$ "), that is, for example if we precisely assume

$$
\forall \delta \leq \delta^{*} \quad \exists\left(y_{\delta}^{o}, v_{\delta}^{o}, \theta_{\delta}^{o}\right) \in \mathcal{D}_{\delta}^{o} .
$$

\section{REFERENCES}

[1] M. Bergounioux, H.Zidani, Pontryagin Maximum Principle for Optimal control of Variational Inequalities, SIAM Journal on Control and Optimization, Vol. 37, $\mathrm{n}^{\mathrm{o}} 4$, pp. 1273-1290, 1999

[2] M. Bergounioux, Optimal Control of an Obstacle Problem, Applied Mathematics and Optimization, 36, pp 147-172 (1997).

[3] J. Burger, M. Pogu, Functional and Numerical Solution of a Control Problem Originating from Heat Transfer, J. Optim. Theo. Appl., Vol. 68 (1991), p. 49-73.

[4] E. Casas, Analisis de la Convergencia en la Approximacion Numerica de Problemas de Control con Restricciones Sobre el Estado, Actas del XII Cedya II Congresso de Matematica Aplicada Oviedo, 1991, p. 301-306.

[5] E. Casas, The Relaxation Theory Applied to Optimal Control Problems of Semilinear Elliptic Equations, in System Modelling and Optimization, J. Dolezal and J. Fidler Eds., Chapman and Hall, 1996, p. 187-194.

[6] G. Choudury, Fully Discrete Galerkin Approximations of Parabolic Boundary-Value Problems with Nonsmooth Boundary Data, Numer. Math., Vol 57(1990), p. 179-203.

[7] G. Choudury, Fully Discrete Galerkin Approximations of Parabolic Boundary-Value Problems with Nonsmooth Boundary Data, Appl. Math. Optim., Vol. 31 (1995), p. 41-55.

[8] P. G. Ciarlet, The Finite Element Method for Elliptic Problems, North Holland, 1978.

[9] K. Erikson, C. Johnson, Adaptive Finite Element Methods for Parabolic Problems IV: A Nonlinear Model Problem, SIAM J. Numer. Anal. Vol. 32 (1995), p. 1750-1763.

[10] I. Lasiecka, Ritz-Galerkin Approximation of the Time Optimal Boundary Control Problem for Parabolic Systems with Dirichlet Boundary Conditions, SIAM J. Cont. Optim., Vol. 22 (1984), p. $477-500$.

[11] U. Mackenroth, Numerical Solution of Some Parabolic Boundary Control Problems by Finite Elements, Control Problems for Systems Described by Partial Differential Equations and Applications, I. Lasiecka and R. Triggiani Eds., Lecture Notes in Control and Information Sciences, Vol. 97 (1987), p. $325-335$. 
[12] P. Neittaanmäki, D. Tiba, Optimal Control of Nonlinear Parabolic Systems, Marcel Dekker Eds., 1994.

[13] R. H. Nochetto, C. Verdi, Approximation of Degenerate Parabolic Problems Using Numerical Integration, SIAM J. Num. Anal., Vol. 25 (1988), p. 784-814.

[14] J. P. Raymond, H. Zidani, Hamiltonian Pontryagin's Principles for Control Problems Governed by Semilinear Parabolic Equations, Applied Mathematics and Optimization

[15] T. Roubicek, A Stable Approximation of a Constrained Optimal Control for Continuous Casting, Num. Funct. Anal. and Optim., Vol. 13 (1992), p. 487-494.

[16] W. Rudin, Analyse réelle et complexe, Masson, Paris (1978).

[17] M. Sibony, Analyse Numérique III, Itérations et Approximations, Hermann, Paris 1988.

[18] V. Thomée Galerkin finite element methods for parabolic problems, Springer Series in Computational Mathematics, Springer-Verlag, Berlin, 1997.

[19] F. Tröltzsch, Semidiscrete Ritz-Galerkin Approximation of Nonlinear Parabolic Boundary Control Problems - Strong Convergence of Optimal Controls, Appl. Math.Optim., Vol. 29 (1994), p. 309329.

[20] C. Verdi, Optimal Error Estimates for an Approximation of Degenerate Parabolic Problems, Numer. Funct. Anal. and Optim., Vol. 9 (1987), p. 657-670.

[21] A. Zenisek, Nonlinear Elliptic and Evolution Problems and their Finite Element Approximations, Academic Press, 1990.

[22] H. Zidani, Optimal Control Problem for Semilinear Parabolic Equation: Optimality Conditions and Numerical Approximations, Thèse, Toulouse, 1996.

[23] J. Zowe and S. Kurcyusz, Regularity and stability for the mathematical programming problem in Banach spaces, Applied Mathematics and Optimization, 5, pp.49-62, 1979. 


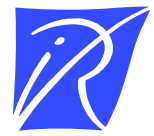

Unité de recherche INRIA Rocquencourt Domaine de Voluceau - Rocquencourt - BP 105 - 78153 Le Chesnay Cedex (France)

Unité de recherche INRIA Lorraine : LORIA, Technopôle de Nancy-Brabois - Campus scientifique 615, rue du Jardin Botanique - BP 101 - 54602 Villers-lès-Nancy Cedex (France)

Unité de recherche INRIA Rennes : IRISA, Campus universitaire de Beaulieu - 35042 Rennes Cedex (France)

Unité de recherche INRIA Rhône-Alpes : 655, avenue de l'Europe - 38330 Montbonnot-St-Martin (France)

Unité de recherche INRIA Sophia Antipolis : 2004, route des Lucioles - BP 93 - 06902 Sophia Antipolis Cedex (France)

INRIA - Domaine de Voluceau - Rocquencourt, BP 105 - 78153 Le Chesnay Cedex (France)

http://www.inria.fr

ISSN 0249-6399 